\title{
Miniaturization of Slot Loop Antenna Using Split-Ring Resonators
}

\author{
Kai-Cheng $\mathrm{Chi}^{1}$, Shih-Yuan $\mathrm{Chen}^{1}$, and Powen $\mathrm{Hsu}^{2}$ \\ ${ }^{1}$ Graduate Institute of Communication Engineering \\ and ${ }^{2}$ Department of Electrical Engineering \\ National Taiwan University, Taipei 10617, Taiwan \\ E-mail: phsu@cc.ee.ntu.edu.tw
}

\section{Introduction}

In recent years, the need for antenna miniaturization stems from the fact that most mobile platforms have a limited space for all of the required antennas in ever increasing wireless systems. Intuitively, substrates with high dielectric constants can be used for miniaturization. However these structures easily excite surface waves, thus degrading the performance of the antenna. Another approach for reducing the antenna size is to change the boundary condition of the antenna, either using a shorting pin on a patch [1] or replacing the two short circuits at the end of the resonant slot by inductive or capacitive loadings [2]. Slow wave concept is also a common approach for miniaturizing the antennas. Using the lump elements with periodical arrangement is presented in [3]. The significant reduction in size is obtained, however at the cost of poor performances. In this paper, we propose a miniaturizing method without using lump elements. In general, Splitring resonators (SRRs) are used to form the left-hand transmission line [4]-[8] and we take advantage of its equivalent inductance and capacitance to achieve the slow wave behavior and reduce the size of the slot loop antenna.

\section{Antenna Design}

The Split ring resonator (SRR) was first proposed in [4]. It consists of two concentric metallic split rings as shown in Fig. 1(a). When excited by a time-varying external magnetic field directed along the $z$-axis, its equivalent circuit can be drawn as that shown in Fig. 1(b), where $L s$ is the self-inductance and $C s$ is the capacitance associated with each SRR half. Using the above LC characteristics of SRR, we propose a miniaturized slot loop antenna with equal slot width by adding the SRRs underneath the slot as shown in Fig. 2 to reduce the antenna size. The magnetic field in the slot loop can excite SRRs and the equivalent circuit of a unit cell of the antenna with size $l$ shown in Fig. 2(b) is shown in Fig. 3. Its dispersion relation is given by [8]

$$
\cos (\beta l)=1-\frac{L a C a \omega^{2}}{2}+\frac{C a / C s^{\prime}}{4\left(1-\omega_{0}^{2} / \omega^{2}\right)}
$$

where $L a$ and $C a$ are the per-section inductance and capacitance of the slot loop antenna, respectively, $C_{s}{ }^{\prime}=L_{s} / \omega_{0}^{2} M^{2}$ is the equivalent capacitance, in which $M$ is the mutual inductance between slot loop and SRR and $\omega_{0}$ is the angular resonant frequency of the SRR. Eq. (1) suggests that for slow waves, the operating frequencies would be lower than the resonant frequency of the SRR. Also, the self inductance $L s$ of SRR is a key parameter to control the percentage of size reduction.

\section{Simulation and Measurement Results}


The proposed antenna is implemented and tested. The dielectric substrate used is FR4 with dielectric constant $\varepsilon_{\mathrm{r}}=4.3$, loss tangent $\tan \delta=0.02$, and thickness $h=0.8 \mathrm{~mm}$. The antenna is matched to a $50-\Omega \mathrm{CPW}$ feedline with $S=4 \mathrm{~mm}$ and $G=0.3 \mathrm{~mm}$. The size of the unit cell of the SRR is $l=6 \mathrm{~mm}$. All other parameters are listed in Table I. The simulated and measured return losses of the slot loop without SRRs are plotted in Fig. 4(a), and those of the slot loop with SRRs are plotted in Fig. 4(b). Good agreement between simulation and measurement is obtained. All simulations in this work were carried out using Ansoft HFSS 10.0. The measured impedance bandwidths (return loss $>$ $10 \mathrm{~dB})$ of the slot loop without and with SRRs are $19.5 \%(3.15-3.83 \mathrm{GHz})$ and $15.8 \%$ $(2.57-3.01 \mathrm{GHz})$, respectively. The slot loop without SRRs loaded shows wider bandwidth than that of the miniaturized design at the expense of a relatively larger antenna size. The size reduction is about $20 \%$ as the resonant frequency being decreased from 3.5 to $2.81 \mathrm{GHz}$. Radiation patterns of the slot loop without and with SRRs measured at 3.5 and $2.81 \mathrm{GHz}$ are shown in Figs. 5 and 6, respectively. Both antennas have slot-dipole-like radiation patterns within their respective bands. The simulated and measured peak gains of the slot loop without SRRs are about 3.02 and $3.95 \mathrm{dBi}$, respectively. After adding the SRRs, the simulated and measured peak gains of the antenna are about 2.83 and $4.53 \mathrm{dBi}$, respectively.

\section{Conclusion}

A CPW-fed slot loop antenna with SRR-loaded has been presented. By adding SRRs underneath the slot loop, miniaturized characteristics can be realized. Simulation and measurement results have shown that the proposed antenna with SRR-loaded have good impedance matching characteristics. Also, the cross polarization in each plane of the pattern is lowered. At the same time, the proposed antenna maintains the value of the gain.

\section{Acknowledgements}

This work was supported by the National Science Council, Taiwan, R.O.C., under Contract NSC 97-2221-E-002-061-MY3.

\section{References}

[1] R. Waterhouse, "Small microstrip patch antenna", Electron. Lett., vol. 31, no. 8, pp. 604-605, Apr. 1995.

[2] R. Azadegan and K. Sarabandi, "A novel approach for miniaturization of slot antennas", IEEE Trans. Antennas Propagat. vol. 51, no. 3, pp. 421-429, Mar. 2003.

[3] P. L. Chi, K. M. Leong, R. Waterhouse, and T. Itoh, "A miniaturized CPW-fed capcitor-loaded slot-loop antenna", Signals, Systems and Electronics, pp. 595-598, Aug. 2007.

[4] J. B. Pendry, A. J. Holden, D. J. Robbins, and W. J. Stewart, "Magnetism for conductors and enhanced nonlinear pheomena", IEEE Trans. Microwave Theory Tech., vol. 47, no. 11, pp. 2075-2084, Nov. 1999.

[5] R. Marques, F. Medina, and R. Rafii-El-Idrissi, "Role of bianisotropy in negative permeability and left-handed metamaterials", Phys. Rev. B, vol. 65, pp.144440-1144440-6, Apr. 2002.

[6] R. Marques, F. Mesa, J. Martel, and F. Medina, "Comparative analysis of edge- and broadside-coupled split ring resonators for metamaterial design-theory and experiments", IEEE Trans. Antennas Propagat., vol. 51, no. 10, pp. 2572-2581, Oct. 2003. 
[7] J. D. Baena, J. Bonache, F. Mesa, R. Marques, F. Falcone, T. Lopetegi, M. A. G. Laso, J. G. Garcia, I. Gil, M. F. Portillo, and M. Sorolla, "Equivalent-circuit models for split-ring resonators for complementary split-ring resonators coupled to planar transmission lines."IEEE Trans. Antennas Propagat., vol. 53, no. 4, pp. 1451-1461, Apr. 2005.

[8] R. Marques, F. Martin, and M. Sorolla, Metamaterials with Negative Parameters, John Wiley \& Sons, 2007, Chaps. 2 and 3.

TABLE I. Design Parameters of The Proposed Antenna (unit: mm)

\begin{tabular}{|c|c|c|c|c|c|c|c|}
\hline$L$ & $L_{1}$ & $L_{2}$ & $r$ & $c$ & $d$ & $t$ & $l$ \\
\hline 50 & 18.75 & 23.5 & 2.75 & 0.3 & 0.3 & 5.5 & 6 \\
\hline
\end{tabular}

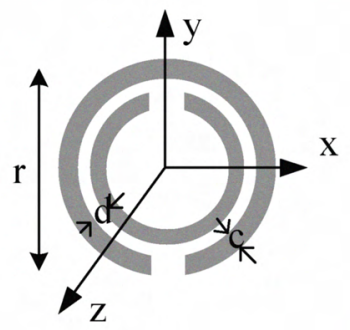

(a)

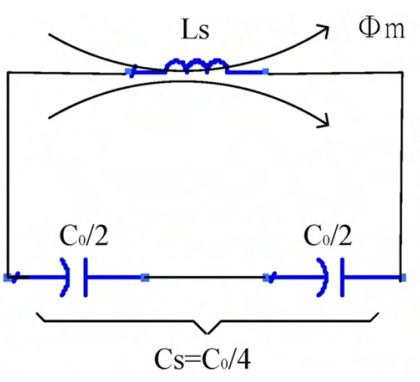

(b)

Fig. 1. (a) Geometry of SRR and (b) its equivalent circuit.
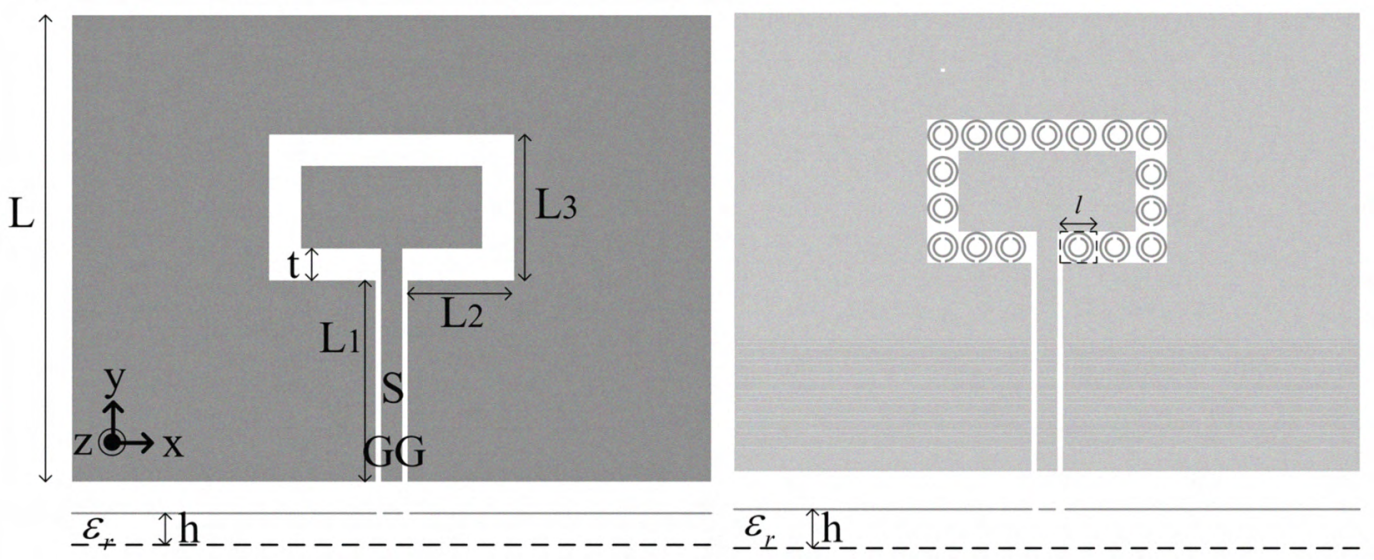

(a)

(b)

Fig. 2. Geometry of the proposed antenna. (a) Top side. (b) Bottom side.

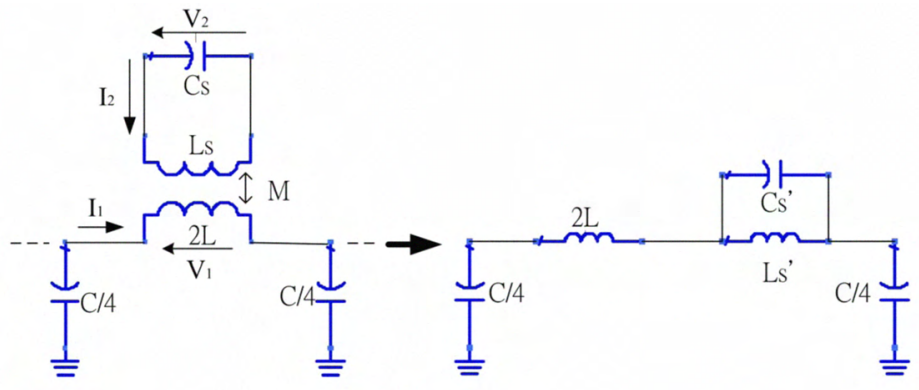

Fig. 3. Equivalent circuit of a unit cell of the slot loop coupled by SRR. 


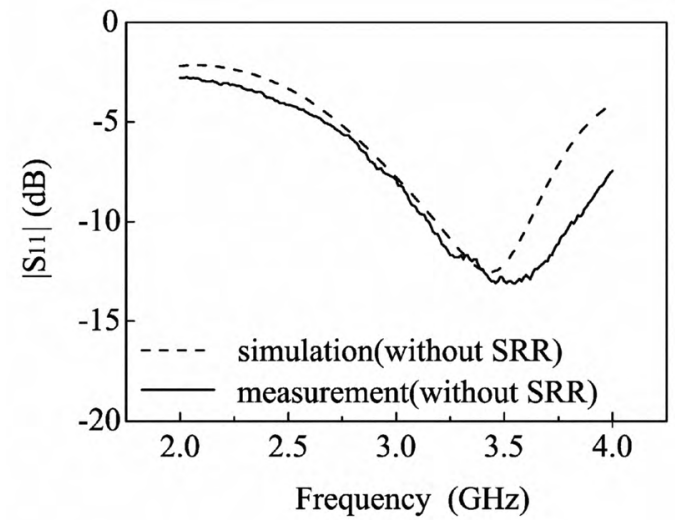

(a)

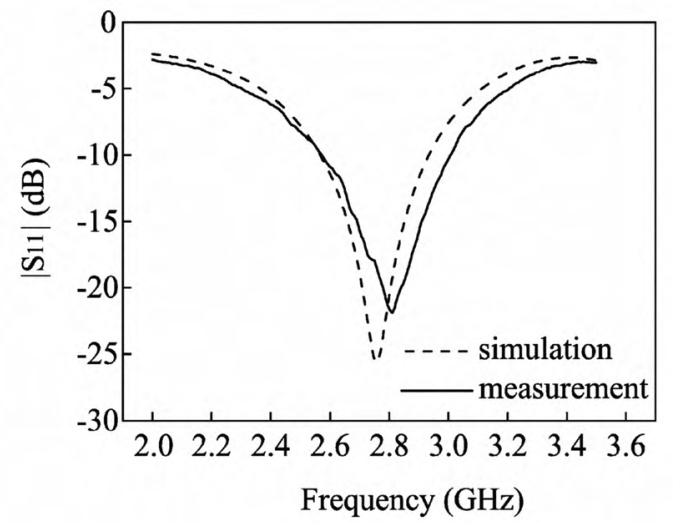

(b)

Fig. 4. Simulated and measured return losses (a) Slot loop without SRRs (b) Slot loop with SRRs.

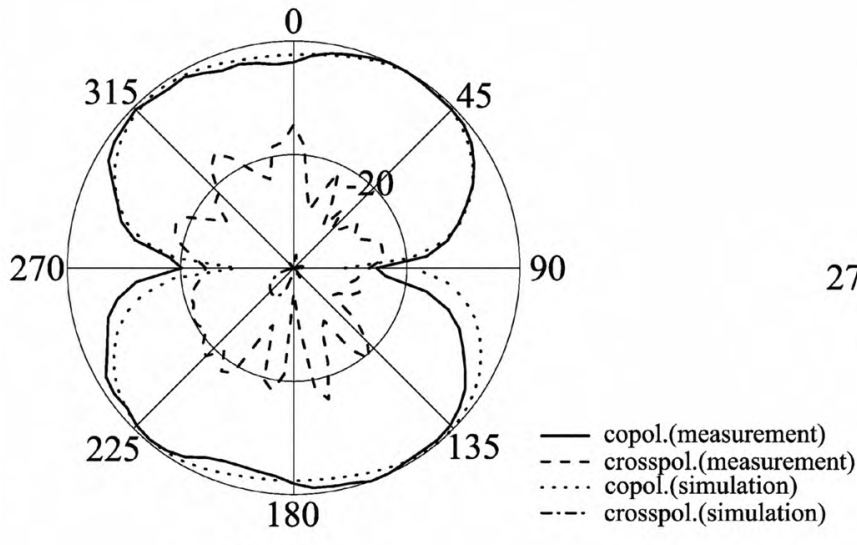

E-plane

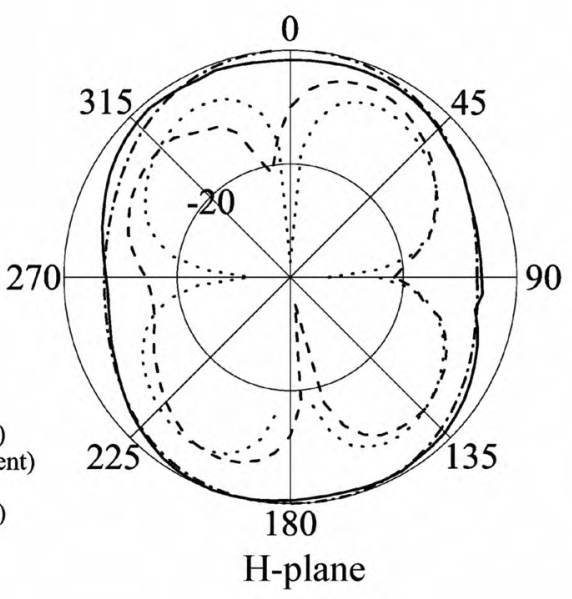

Fig. 5. Measured and simulated radiation patterns of the slot loop without SRRs at 3.5 GHz.

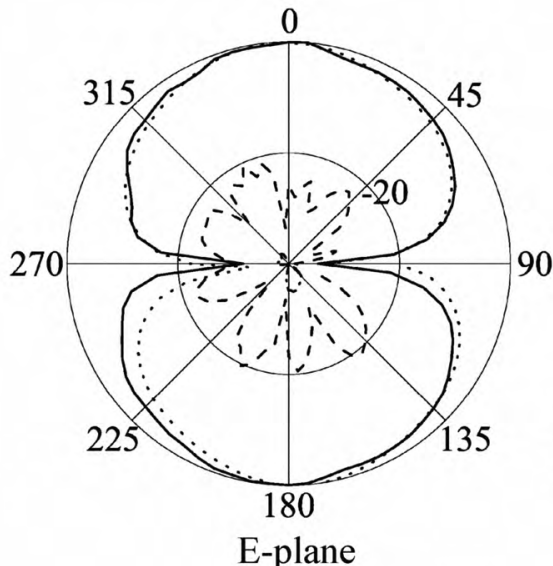

E-plane

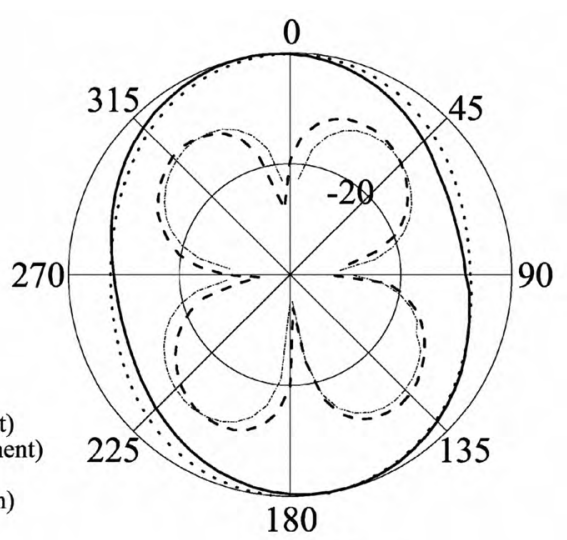

H-plane

Fig. 6. Measured and simulated radiation patterns of the slot loop with SRRs at $2.81 \mathrm{GHz}$. 